\title{
The expression of NLK is functionally associated with colorectal cancers (CRC)
}

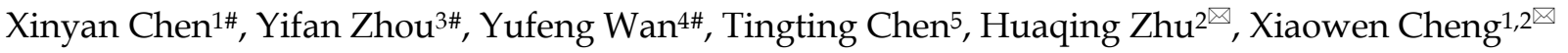 \\ 1. Department of Clinical Laboratory, The First Affiliated Hospital of Anhui Medical University, Hefei, Anhui 230022, P.R. China. \\ 2. Laboratory of Molecular Biology and Department of Biochemistry, Anhui Medical University, Hefei, Anhui 230022, P.R. China. \\ 3. Department of Pathology, The First Affiliated Hospital of USTC, Division of Life Sciences and Medicine, University of Science and Technology of China, Hefei, Anhui, \\ 230036, China. \\ 4. Department of Otolaryngology, The Affiliated Chaohu Hospital of Anhui Medical University, Hefei, Anhui 238001, P.R. China. \\ 5. Department of Pathology, Anhui Medical University, Hefei, Anhui 230032, P.R. China. \\ \#These authors contributed equally to this work.
}

$\triangle$ Corresponding authors: Dr Xiaowen Cheng, Department of Clinical Laboratory, The First Affiliated Hospital of Anhui Medical University,Hefei, Anhui 230022, P.R. China. E-mail: chengxiaowen@ahmu.edu.cn; or Dr Huaqing Zhu, Laboratory of Molecular Biology and Department of Biochemistry, Anhui Medical University, 81 Meishan Road, Hefei, Anhui 230032, P.R. China. E-mail: aydzhq@126.com.

(C) The author(s). This is an open access article distributed under the terms of the Creative Commons Attribution License (https://creativecommons.org/licenses/by/4.0/). See http://ivyspring.com/terms for full terms and conditions.

Received: 2021.05.10; Accepted: 2021.10.01; Published: 2021.10.17

\begin{abstract}
The regulatory mechanism of NLK in the carcinomagenesis and progression of colorectal cancer (CRC) remains unclear. Here, we identified a single nucleotide polymorphism (SNP) site of NLK (rs2125846) as a new susceptibility locus for CRC risk located within an intron of the human NLK gene in a Chinese population. NLK downregulation led to a decrease in the ability of proliferation and migration of RKO cells in vitro. The proportion of RKO apoptotic cells increased by interfering with the endogenous expression of NLK. We speculate that LncRNA XIST may upregulate NLK expression by downregulating miR-92b-3p, thereby promote the development of CRC. These results provide important information for the identification of novel potential targets for the prevention or treatment of CRC.
\end{abstract}

Key words: colorectal cancer, NLK, single nucleotide polymorphism, LncRNA XIST, microRNA-92b-3p

\section{Introduction}

Colorectal cancer $(\mathrm{CRC})$ is one of the most common malignant cancers worldwide. CRC treatments, including surgical resection, radiotherapy, and chemoradiotherapy, have improved in recent years. Statistics show that $50 \%$ of patients with stage III can be cured by surgery, $20 \%$ of patients will survive following adjuvant chemotherapy, and $30 \%$ of patients will relapse within 2-3 years. Therefore, only $20 \%$ of patients with stage III benefit from chemotherapy, leaving $80 \%$ exposed to unnecessary toxicity [1]. Moreover, the prognosis of CRC patients is based on their clinicopathological features, mainly focusing on the stage at diagnosis. The five-year overall survival rate is $\sim 90 \%$ for the first stage and then decreases to $70 \%, 58 \%$, and less than $15 \%$ for the second, third, and fourth stages, respectively. Many studies have focused on searching for well-known oncogenes or tumor-suppressor genes in CRC. Significant technological developments in the field of molecular biology have been used in clinics to detect gene expression in tumor tissue samples. Owing to the limited predictive value, high cost, and unconventional use of some clinical molecular markers and the lack of clear treatment guidance, selecting the most effective therapy for CRC patients remains a challenge. Therefore, identifying and exploring new molecular indicators is a topic of high interest [2].

Approximately $35 \%$ of CRC risk can be attributed to genetic factors [3]. Single nucleotide polymorphisms (SNPs) constitute more than $90 \%$ of the genetic variation in the human genome. SNPs can lead to abnormal cell differentiation and promote tumor development. In the past 10 years, more than 60 SNPs have been found to be associated with CRC [4]. Nemo-like kinase (NLK) is an evolutionarily conserved mitogen-activated serine/threonine protein kinase [5]. In 1994, it was first reported that 
nemo gene mutation decreased the survival of Drosophila and led to abnormal head and eye development. The mammalian nemo homolog was cloned in 1998 and named NLK [6]. Extensive evidence shows that abnormal NLK expression is closely related to the carcinomagenesis and progression of human cancers. It is highly expressed in laryngeal carcinoma, lung cancer, and cervical squamous cell carcinoma [7-9] but remains at low levels in non-small cell lung cancer, breast cancer, and ovarian cancer [10-12]. However, the contribution of SNPs to the expression of NLK in relation to the risk to $\mathrm{CRC}$ is much less clear. Previous studies analyzing more than 1000 samples and nearly 400 SNP sites in 67 mitotic kinases found that rs2125846 in the NLK gene is associated with an increased risk of ovarian cancer [13]. In this case-control study, we analyzed the relationship between this NLK SNP and the risk of CRC in a Chinese population to provide a reference for the prevention and treatment of CRC in high-risk groups. However, the expression change of NLK in $\mathrm{CRC}$ is still controversial. Li et al analyzed the protein expression of NLK in CRC tissues by immunohistochemical (IHC) analysis and found that its expression was increased in colorectal cancer tissues compared with adjacent normal tissues $[14,15]$. Han et al found that the mRNA level of NLK in CRC tissues was lower than that in adjacent tissues by RT-PCR [16]. In this study, we detected the expression of NLK in CRC tissues and observed that changes in NLK levels affected the biological behavior of CRC cells at the cellular level.

The genome sequencing project has revealed that more than $90 \%$ of genomes are transcribed into non-coding RNA (ncRNA), and only $2 \%$ of the human genome consists of protein-coding genes [17]. With the recent development of functional genomics, ncRNA has become a primary focus of gene regulation research. Long-stranded non-coding RNA (LncRNA) and microRNA (miRNA) are the two most intensively studied ncRNAs [18]. Increasing evidence shows that the occurrence and progression of many diseases, including cancer, are related to an imbalance in the regulation of miRNAs and LncRNAs $[19,20]$. In 2009, Seitz et al [21] proposed that most RNA transcripts with miRNA response elements (ncRNAs) can act as competitive inhibitors of miRNA. RNAs involved in this process are termed miRNA sponges, and they regulate the expression and function of miRNA by competing with endogenous mRNA for miRNA binding sites. In 2011, Salmena et al. [22] proposed the competitive endogenous RNA (ceRNA) hypothesis. They speculated that ncRNAs, especially LncRNAs, can act as endogenous miRNA sponges to inhibit miRNA function, thus affecting diverse
miRNA targets. This interaction can decrease the level and activity of miRNAs. In recent years, ncRNAs, including microRNAs and LncRNAs, have been increasingly suggested as a new class of clinical biomarkers and potential targets for cancer therapy [23].

LncRNAs participate in the development of CRC by coordinating with miRNAs and protein-coding mRNAs. LncRNAs modify the expressions of targeted genes by competinging with miRNAs for the binding sites on the mRNAs. Recent studies have shown that regulatory networks formed by the LncRNA/ miRNA/mRNA interactions regulate the initiation and development of CRC [24]. miRNA-92b-3p (miR-92b-3p) directly targets NLK in oral squamous cell carcinoma [25] and glioma [26], and lncRNA XIST directly acts on miR-92b-3p in hepatoma cells [27]. Therefore, via analyzing the effect of NLK on the CRC cells, we further explored the roles of LncRNAs and miRNAs in regulating the expression of NLK. More specifically, we addressed the relationships among XIST, miR-92b-3p, and NLK in CRC first by investigating the expressions of XIST and miR-92b-3p in the CRC and normal colorectal tissues, and by analyzing the effect of the XIST/miRNA-92b-3p/NLK signaling axis on the carcinomagenesis and progression of CRC, using a dual luciferase reporter system and by silencing XIST and miR-92b-3p.

In this study, we explored the possible roles of NLK in the carcinomagenesis and progression of CRC from clinical perspectives with hope to provide meaningful implications for searching the markers for early diagnosis as well as drug targets for CRC treatment.

\section{Materials and methods}

\section{Patients and samples}

147 CRC cases and 150 healthy subjects were enrolled in the present study. Healthy participants who matched the age and sex of the case group were randomly selected as the control group. Thirty-eight fresh CRC tissue pairs (tumor and adjacent normal tissues) were obtained by surgical resection from the First Affiliated Hospital of Anhui Medical University and immediately stored in RNAlater at $-80{ }^{\circ} \mathrm{C}$. All cases were diagnosed by histopathology, and patients with preoperative radiotherapy and chemotherapy or with autoimmune diseases such as diabetes and nephropathy were excluded. The tissue wax blocks, and sections of CRC were obtained from the Department of Pathology of the First Affiliated Hospital of Anhui Medical University and confirmed via histopathological diagnosis by pathologists. Informed consent was obtained from each enrolled 
patient. The study protocols were approved by the Ethics Committee of Anhui Medical University.

\section{DNA extraction}

DNA was extracted from peripheral blood leukocytes according to the manufacturer's instructions of the DNA extraction kit (Baiao Company, Shanghai). The content and purity of DNA were detected using a NanoDrop One/OneC micronucleic acid protein concentration analyzer (Thermo, USA). The ratio of the absorbance at 260 and $290 \mathrm{~nm}$ was between 1.8 and 2.0.

\section{SNP genotyping}

The high-resolution melting (HRM) method was used to determine the SNP genotypes. HRM statistics were collected from the Roche LightCycler96 fluorescence quantitative PCR instrument. The primers used were as follows: NLK-SNP-F, GCTCTA ACTTCTAATGGAAGTC and NLK-SNP-R, CAGATC CATACATGAGAAACAC.

\section{Immunohistochemistry (IHC)}

Tissue sections were deparaffinized, rehydrated, and heated using citrate antigen repair solution under high pressure for antigen retrieval. Then, the slides were incubated with 3\% hydrogen peroxide at room temperature to block endogenous peroxidase activity and subsequently incubated with the appropriate amount of a polyclonal anti-NLK antibody (Cell Signaling Technology, Beijing) for 3 hours at room temperature. The sides were washed three times with PBS, followed by incubation with the second antibody and and treated with DAB for 5 minutes. The slides were examined under a microscope, and the results were analyzed with Image J software to calculate the positive IHC signals.

\section{TdT-mediated dUTP Nick-End Labeling (TUNEL)}

A one-step TUNEL apoptosis detection kit (blue skies, Shanghai) was used to determine the rate of apoptosis in CRC tissues and adjacent normal tissues. After dewaxing in water, $20 \mu \mathrm{g} / \mathrm{ml}$ of DNase-free proteinase $\mathrm{K}$ was added for 30 minutes. The TUNEL detection solution (fluorescence labeling solution:TdT enzyme $=9: 1$ ) was prepared and incubated with tissue sections for 30 minutes. The slides were washed three times with PBS, and DAPI dye solution was added for 3 minutes. After washing with PBS three times, an anti-fluorescence quencher was added dropwise. The slides were mounted and later observed under a microscope.

\section{Cell culture and transfection}

The human CRC cell line RKO was purchased from the American Type Culture Collection (USA). It was then cultured in Dulbecco's Modified Eagle Medium (Hyclone, Beijing, China) with 10\% fetal bovine serum (FBS) and incubated in a humidified incubator with $5 \% \mathrm{CO} 2$ at $37{ }^{\circ} \mathrm{C}$. The NLK overexpression lentivirus vector NLK-LV5 (EF-1aF/ GFP\&Puro) and NLK interference lentivirus vector NLK-shRNA- LV3 (H1/GFP\&Puro) were obtained from Shanghai Jima Pharmaceutical Technology Co. Ltd. For cell transfections, RKO cells were seeded into a 12-well plate, cultured overnight, and transfected with an appropriate amount of lentivirus containing NLK-LV5, negative control (LV5NC), NLK-shRNALV3, or negative control (LV3NC). The medium was replaced with fresh medium after 12 hours. The state of the cells and the proportion of GFP expression were observed daily. All transfects were tested regularly by qRT-PCR and western blot to ensure the efficiency of overexpression or knockdown. The target sequences of NLK shRNA were as follows: NLK-KD, 5'-GGATA GACCTATTGGATATGG-3' and LV3NC, 5'-TTCTCC GAACGTGTCACGT-3'.

\section{Cell proliferation assay}

The proliferation rate of RKO cells was measured with the Cell Counting Kit-8 (CCK-8; 7sea, Shanghai) assay and colony formation assay. For the cell count assay, control and transfected cells were cultured in a 96-well plate (2000 cells/well) and incubated at $37{ }^{\circ} \mathrm{C}$ in $5 \% \mathrm{CO} 2$. Triplicate wells were measured in each group. After adding $10 \mu \mathrm{l} \mathrm{CCK-8}$ solution to each well, the plate was incubated for 2 hours. Then, the spectrophotometric absorbance was measured at a wavelength of $450 \mathrm{~nm}$ using a microplate reader (Thermo Scientific, USA) for each sample. For the colony formation assay, control and transfected cells were seeded into a six-well plate (400 cells/well) and incubated at $37^{\circ} \mathrm{C}$ in $5 \%$ CO2 for $8-10$ days. Triplicate wells were measured in each group. Cell culture was terminated when visible clones were observed, and then the colonies were stained with crystal violet. The colonies were carefully washed with $\mathrm{ddH} 2 \mathrm{O}$ three times, and images were taken after drying. The number of colonies was counted three times, the average value was obtained, and the number of clones was compared among the groups.

\section{Cell migration assay}

The cell migration assay was performed in a Transwell chamber (8- $\mu \mathrm{m}$ pore size; CORNING). $5 \times 10^{4}$ cells in $100 \mu \mathrm{l}$ serum-free DMEM was added to the top chamber, and $600 \mu \mathrm{l}$ of medium containing $10 \%$ FBS were placed into the bottom chamber as an attractant. The non-migrated cells were removed from the top chamber after incubation for $24 \mathrm{~h}$. The 
chambers were then fixed with a $4 \%$ methanol solution, stained with crystal violet, and photographed.

\section{Flow cytometry detection}

Flow cytometry was used to detect the apoptosis and cell cycle phase of CRC cells. Annexin V-PE and 7-ADD double staining (BD Biosciences, America) was performed in accordance with the manufacturer's instructions to detect cell apoptosis. Then, $200 \mu \mathrm{l}$ 1X Annexin V Binding Buffer was added to the solution, and the cells were analyzed using a flow cytometer. For the cell cycle assay, RNase A and PI staining solutions were added to collected cells in accordance with the supplier's instructions (BeiBo, Shanghai). Then, the cell cycle was detected using a flow cytometer.

\section{Western blot}

Briefly, the harvested cells were washed with PBS and lysed with $1 \times$ SDS lysis buffer. The BCA method was used to measure the protein concentration. Samples were separated by $10 \%$ SDS-PAGE and transferred to the PVDF membrane. The membranes were blocked with $5 \%$ fat-free milk in PBS, $0.1 \%$ Tween 20 for 2 hours at room temperature, followed by overnight incubation with the primary antibody at $4{ }^{\circ} \mathrm{C}$. Next, the secondary antibody was incubated for 1 hour at room temperature, and the protein bands were detected using the ECL reaction solution.

\section{Quantitative real-time polymerase chain reaction (qRT-PCR)}

Total RNA was extracted from cultured cells using a total RNA extraction kit (TIANGEN, Beijing) in accordance with the manufacturer's instructions. Then, cDNA synthesis was performed using a reverse transcription kit (TAKARA, Dalian) following the manufacturer's instructions. A fluorescent quantitative PCR kit (TAKARA, Dalian) was used for qPCR analysis. The relative gene expression level was calculated by the $2 \Delta \mathrm{Ct}$ method. All experiments were repeated three times, and GAPDH was used as the internal control. The following primers were used: NLK: F: 5'-TATCGGGCTCCAGAAATCCT-3', and R: 5'-GTGTGCCCAACAGATCCGT-3'; LncRNA XIST: F: 5'-GAGGCAAGATGGATGATAGCAG-3', and R: 5'-ACAATCACGCAAAGCTCCTAAC-3'; miR-22-3p: 5'-CTGCTATTGCACTCGTCCC-3'; U6: F: 5'-CTCGC TTCGGCAGCACA-3', and R: 5'-AACGCTTCACGA ATTTGCGT-3'.

\section{Dual-luciferase reporter gene assay}

The 293T cells were cultured for co-transfections. The XIST and NLK 3'-UTR regions, containing potential miR-92b-3p binding sites, were predicted using TargetScan version 7.11. The predicted 3'-UTR fragments were amplified by PCR. Mutants were then constructed by introducing point mutations into the seed binding site for miR-92b-3p. The wild type and mutant fragments (wt-Luc-XIST and wt-Luc-NLK, and mu-Luc-XIST and mu-Luc-NLK) were subcloned into the psiCHECK2 vector (Promega Corporation, United States), downstream of the renilla luciferase gene. The vector also contains the firefly luciferase gene. Cells were seeded in 24-well plates and cotransfected with wild-type or mutated luciferase constructs along with miR-92b-3p mimics, miR92b-3p inhibitors, or controls. The Dual Luciferase Reporter Assay System (Promega) was used $48 \mathrm{~h}$ after transfection following the manufacturer's protocol. The relative luciferase activity was calculated using the ratio of firefly luciferase activity to renilla luciferase activity.

\section{Statistical analysis}

SPSS20.0 software was used for analysis. The results were subjected to one-way ANOVA and parametric $t$-testing and were expressed as the mean \pm SD. An unconditional logistic regression model was used to analyze the association between different genetic models and $\mathrm{CRC}$, and the odds ratio (OR) and $95 \%$ confidence interval $(95 \% \mathrm{CI})$ were calculated. The AA genotype represents the homozygous wild type, the AG genotype represents the heterozygous mutation, and the GG genotype represents the homozygous mutation. The genetic models used in this study included a codominant genetic model [polymorphic homozygous vs wild-type homozygous, and heterozygous vs wild-type homozygous--(GG/AA and AG/AA)], dominant genetic model [heterozygous + polymorphic homozygous vs wild-type homozygous--(GG+AG)/ $\mathrm{AA}$, and recessive genetic model [polymorphic homozygous vs wild-type homozygous + heterozygous --GG/(AA+AG)]. The goal of this analysis using the model referred is to determine risk factors for CRC. A logistic regression model was used to analyze the interaction between multiplicative models. Statistical significance was accepted at a level of $P$ value $<0.05$.

\section{Results}

\section{NLK-rs2 125846 (A/G) genotypes correlates with the susceptibility to CRC}

This case-control study enrolled 147 CRC patients and 150 healthy subjects. The two groups were comparable and no significant difference in sex or age was found (Table 1). 

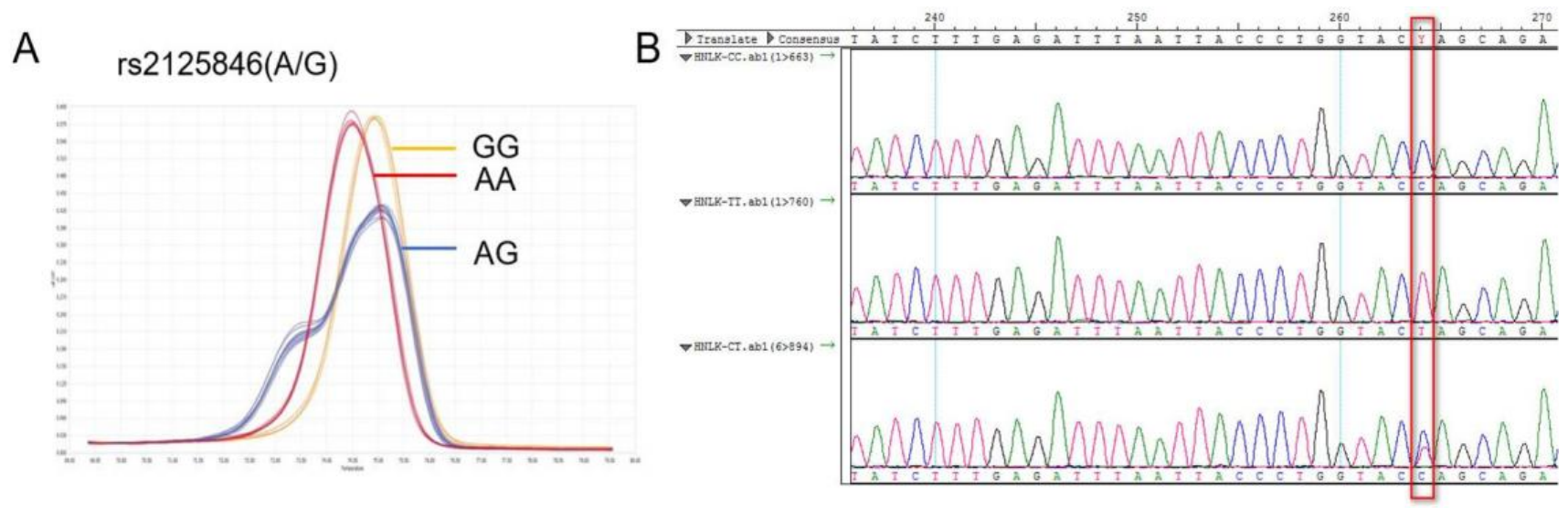

Figure 1. NLK rs2125846 A / G HRM curve (A) and sequencing map (B)

Table 1. Comparison of general information between patient group and normal group

\begin{tabular}{lllll}
\hline Group & Patient $(\mathrm{n}=147)$ & Normal $(\mathrm{n}=150)$ & $\mathrm{X}^{2} / \mathrm{t}$ & P value \\
\hline $\begin{array}{l}\text { Gender [n (\%)] } \\
\text { male }\end{array}$ & $86(49.1)$ & $89(50.9)$ & 0.021 & 0.884 \\
female & $61(50.0)$ & $61(50.0)$ & & \\
Age & $59.32 \pm 11.23$ & $57.24 \pm 10.12$ & 1.677 & 0.095 \\
\hline
\end{tabular}

The NLK gene is located at human chromosome $17 \mathrm{p} 12$, rs2125846 is located at the $6^{\text {th }}$ intron $(2364 \mathrm{~A} / \mathrm{G})$ of the NLK gene. An A-G base substitution was identified at position 2364 as follows, GAGAAACACTTTTATGATGTCTGCT[A/G]GTAC CAGGGTAATTAAATCTCAA. The reverse mutation was $A / G$, and the forward mutation was $T / C$ (Figure $1)$.

Rs2125846 was characterized by AA, AG, and GG genotypes, and its distribution in the control and case groups was in accordance with Hardy-Weinberg genetic balance $(\mathrm{P}>0.05)$. In the case group, the AA genotype accounted for $44.1 \%$, AG for $52.4 \%$, and GG for $3.5 \%$ of the population, whereas in the control group, the figures were $46.7 \%, 42.0 \%$, and $11.3 \%$ $(\mathrm{P}<0.05)$. Therefore, the rs2125846 polymorphism exhibited distinct patterns among the case group and the normal group (Table 2).

Table 2. The correlation between genotype distribution of NLK rs2125846 and cancer risk

\begin{tabular}{llllll}
\hline Groups & AA [n (\%)] & AG [n (\%)] & GG [n (\%)] & $\chi^{2}$ value & P value \\
\hline normal & $70(46.7)$ & $63(42.0)$ & $17(11.3)$ & 7.79 & 0.02 \\
patient & $63(44.1)$ & $75(52.4)$ & $5(3.5)$ & & \\
\hline
\end{tabular}

Using the co-dominant genetic model, no significant difference in the CRC susceptibility was observed between the AA genotype and the AG genotype groups $(P>0.05) \quad(\mathrm{OR}=0.327, \quad 95 \% \mathrm{CI}$ : 0.114-0.937). Furthermore, the risk of CRC in patients with the GG genotype was 0.327 times that of the AA genotype. In the dominant genetic model, the AA genotype was considered the control group, and the corresponding $\mathrm{P}$ value for GG/AG was 0.654, indicating no significant difference. In the recessive genetic model, compared with the GG genotype, the G-A substitution that results in the AA/AG phenotypes was more closely associated with the onset of CRC (3.528-fold) (Table 3).

Table 3. Genotype distribution and allele frequency of NLK rs2125846 in cases and controls and analysis of unconditional logistic regression model

\begin{tabular}{|c|c|c|c|c|}
\hline & Normal [n (\%)] & Patient $[\mathrm{n}(5)]$ & OR $(95 \% \mathrm{CI})$ & $P$ value \\
\hline \multicolumn{5}{|c|}{ Co-dominant genetic model } \\
\hline AA & $70(46.7)$ & $63(44.1)$ & 1 & \\
\hline AG & $63(42.0)$ & $75(52.4)$ & $1.323(0.821,2.132)$ & 0.251 \\
\hline GG & $17(11.3)$ & $5(3.5)$ & $0.327(0.114,0.937)$ & 0.037 \\
\hline \multicolumn{5}{|c|}{ Dominant genetic model } \\
\hline AA & $70(46.7)$ & $63(44.1)$ & 1 & \\
\hline $\mathrm{GG}+\mathrm{AG}$ & $80(53.3)$ & $80(55.9)$ & $1.111(0.701,1.761)$ & 0.654 \\
\hline \multicolumn{5}{|c|}{ Recessive genetic model } \\
\hline GG & $17(11.3)$ & $5(3.5)$ & 1 & \\
\hline $\mathrm{AA}+\mathrm{AG}$ & $133(88.7)$ & $138(96.5)$ & $3.528(1.266,9.834)$ & 0.016 \\
\hline
\end{tabular}

The rs2125846 polymorphism was further analyzed in correlation to common clinicopathological features, age of the patients, main location of CRC, drinking history, and family history of the study groups. As shown in Table 4, the polymorphism at this locus was closely related to tumor size, tissue infiltration depth, and drinking history $(P<0.05)$ as opposed to tissue differentiation, tissue typing, age, tumor location, distant metastasis, TMN, and family history $(P>0.05)$ (Table 4$)$.

\section{NLK was upregulated in CRC}

The expression of NLK were investigated first using IHC. There was a clear increase in the level of NLK in clinical CRC tissues compared with adjacent tissues (Figure 2A), which was also statistically significant (Figure 2B) and was further reflected at 
transcriptional level when analyzed by qPCR $(P<0.05)$ (Figure 2C). The proportion of apoptotic cells, revealed by the TUNEL assay, was doubled $(\sim 30 \%)$ in the adjacent tissue compared to the one in the CRC tissues $(\sim 15 \%)$, suggesting an anti-apoptotic role by NLK (Figure 2D).

\section{NLK downregulation decreased the proliferation, colony forming, and migration abilities of RKO cells}

NLK knockdown in the CRC cell lines such as HT-29 and HCT116 affected cell proliferation, colony formation, migration ability, and led to cell cycle arrest at G1/S phase [15, 16]. Previous study had designated four main CRC subtypes based on gene expression profiles and summarized as the consensus molecular subtypes (CMS). According to the study, the RKO and HCT116 cell lines were described as CMS1 subtypes, defined by hypermutation, MSI, and strong immune activation, while HT29 cell line was less likely to be appropriate models for CRC tumors. Moreover, result from other study shows that RKO and HT29 are KRAS wild-type cells, while HCT119 cells carry G13D point mutations. And another study shows that RKO and HCT116 cell lines have no TP53 mutation identified DHPLC analysis [28-30]. These evidences indicate the differences between RKO cells and other two cell lines. Based on the effect of NLK knockdown on the phenotype of the CRC cell line RKO remains unknown, RKO cells were selected for the following experiments to address this question.
A

BLANK

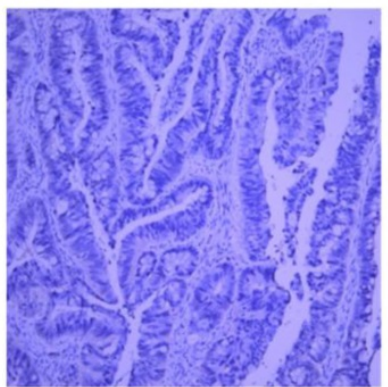

B

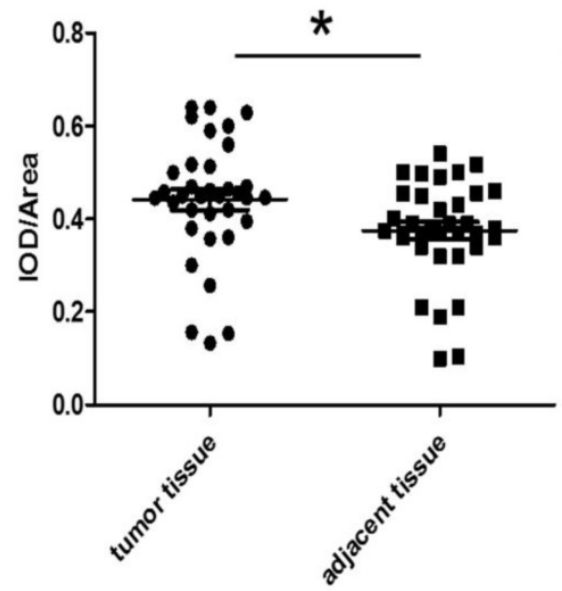

TUMOR TISSUE
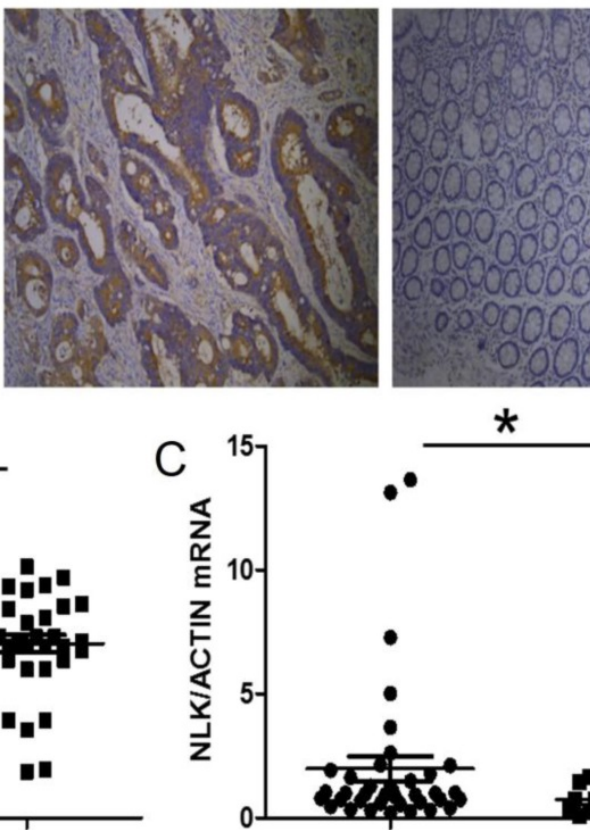

\section{ADJACENT TISSUE}

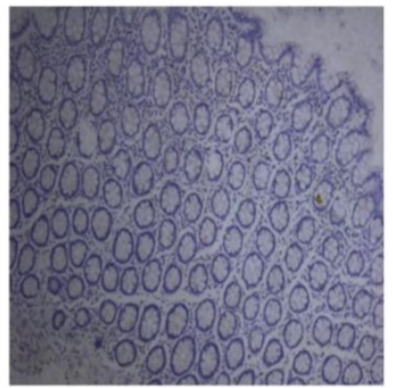

*

D

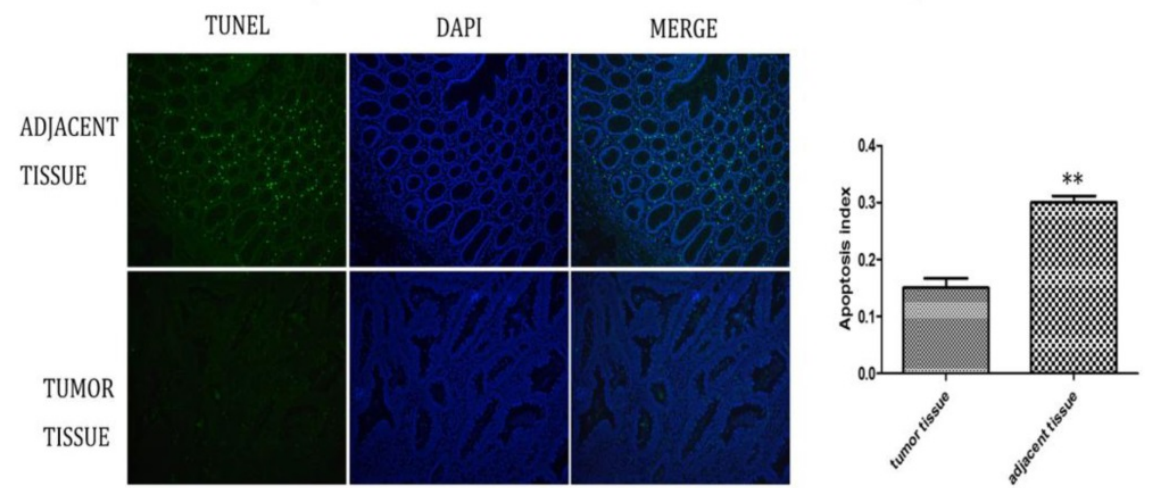

Figure 2. NLK was upregulated in CRC tissues. (A and B) Tumor sections stained with DAB, and NLK expression was examined by immunohistochemistry. Magnification, 100×. $* P<0.05$. (C) The mRNA levels of NLK were detected by qRT-PCR in tumor tissues and adjacent tissues. $* P<0.05$. (D) TUNEL assay of CRC and adjacent tissues. Magnification, 100×. $* * P<0.01$. TUNEL, TdT-mediated dUTP Nick-End Labeling. 
Table 4. Relationship between clinicopathological features and NLK 2125846 genotype polymorphism

\begin{tabular}{|c|c|c|c|c|}
\hline Analytical index & AA & AG & $\chi^{2}$ & $P$ value \\
\hline \multicolumn{5}{|l|}{ Pathological feature } \\
\hline \multicolumn{5}{|l|}{ Diameter } \\
\hline$<5$ & $0(0.0)$ & $6(8.2)$ & $-\mathrm{a}$ & 0.033 \\
\hline$\geq 5$ & $59(100.0)$ & $67(91.8)$ & & \\
\hline \multicolumn{5}{|c|}{ Tissue differentiation } \\
\hline Low & $6(10.7)$ & $15(20.8)$ & 2.352 & 0.125 \\
\hline Middle & $50(89.3)$ & $57(79.2)$ & & \\
\hline \multicolumn{5}{|l|}{ Tissue typing } \\
\hline Ulcerative & 45 (76.3) & $59(80.8)$ & 0.404 & 0.525 \\
\hline Uplift type & $14(23.7)$ & $14(19.2)$ & & \\
\hline \multicolumn{5}{|l|}{ Age } \\
\hline$<50$ & $16(25.4)$ & $14(18.7)$ & 1.839 & 0.399 \\
\hline $50-60$ & $15(23.8)$ & $25(33.3)$ & & \\
\hline$>60$ & $32(50.8)$ & $36(48.0)$ & & \\
\hline \multicolumn{5}{|l|}{ Tumor site } \\
\hline colon & $23(36.9)$ & $32(42.7)$ & 0.542 & 0.462 \\
\hline rectum & $40(63.5)$ & $43(57.3)$ & & \\
\hline \multicolumn{5}{|l|}{ Infiltration depth } \\
\hline $\mathrm{T} 1$ & $4(6.8)$ & $2(2.7)$ & 12.824 & 0.005 \\
\hline $\mathrm{T} 2$ & $11(18.6)$ & $20(27.4)$ & & \\
\hline T3 & $7(11.9)$ & $24(32.9)$ & & \\
\hline $\mathrm{T} 4$ & 37 (62.7) & $27(37.0)$ & & \\
\hline \multicolumn{5}{|c|}{ Lymph node metastasis } \\
\hline N0 & $34(57.6)$ & $37(50.7)$ & $-\mathrm{a}$ & 0.833 \\
\hline N1 & $18(30.5)$ & $26(35.6)$ & & \\
\hline N2 & $7(11.9)$ & $9(12.3)$ & & \\
\hline $\mathrm{Nx}$ & $0(0.0)$ & $1(1.4)$ & & \\
\hline \multicolumn{5}{|l|}{ Distant metastasis } \\
\hline M0 & 59 (93.7) & $73(97.3)$ & 1.116 & 0.262 \\
\hline M1 & $4(6.3)$ & $2(2.7)$ & & \\
\hline \multicolumn{5}{|l|}{ TNM Stage } \\
\hline I & $13(20.6)$ & $14(18.7)$ & 2.225 & 0.531 \\
\hline II & $20(31.7)$ & $25(33.3)$ & & \\
\hline III & $25(39.7)$ & $34(45.3)$ & & \\
\hline IV & $5(7.9)$ & $2(2.7)$ & & \\
\hline \multicolumn{5}{|l|}{ Drinking history } \\
\hline No & $58(92.1)$ & $57(76.0)$ & 6.631 & 0.012 \\
\hline Yes & $5(7.9)$ & $18(24.0)$ & & \\
\hline \multicolumn{5}{|l|}{ Family history } \\
\hline No & $62(98.4)$ & $69(92.0)$ & 2.924 & 0.087 \\
\hline Yes & $1(1.6)$ & $6(8.0)$ & & \\
\hline
\end{tabular}

An ectopic copy of NLK was introduced into RKO cells using a lentivirus vector (NLK-LV5) with the empty vector (LV5 NC) as control. The RNA interference (RNAi) specifically targeting NLK expression was achieved with a lentivirus vector (NLK-shRNA-LV3). The overexpression (OE) lentiviral vector NLK-LV5 (EF-1aF/GFP\&Puro) and the RNAi lentiviral vector NLK-shRNA-LV3 (H1/GFP\&Puro) were used to infect RKO cells, and then the OE and RNAi stable cell lines were constructed by puromycin selection and flow sorting. The empty backbone vector was used as control for $\mathrm{OE}$ and a scrambled shRNA-containing vector as control for RNAi. Both qPCR and Western blot results confirmed the altered expressions of NLK as intended (Figure 3A). The cell proliferation, measured with
CCK-8 test and the colony forming assay, appeared significantly suppressed by the RNAi but was moderately upregulated with NLK overexpression. Compared with the corresponding OE control, NLK overexpression leads to an increase in the proliferation rate of RKO cells and in the number of clone formation. Compared with the corresponding RNAi control, the inhibition of NLK expression results in a decrease in the cell proliferation speed of RKO cells and in the number of clone formation (Figure 3B \& 3C). Furthermore, the trans-well migration test revealed that NLK RNAi severely compromised the cell migration, in contrast to the promoted migration occurred with NLK overexpression (Figure 3D).

\section{NLK knockdown promoted the RKO cell apoptosis and $\mathbf{G} \mathbf{I}$ phase cell cycle arrest of RKO cells}

Following the clue to the potential link between apoptosis and NLK expression, we used flow cytometry (FACS) to examine the apoptosis and cell cycle of RKO cells upon altering NLK expression. The size of apoptotic cell population was significantly reduced with NLK overexpression (OE) compared with the control, in contrast to the scenario in the NLK-deficient RKO cells where the apoptosis was greatly enhanced (Figure 4A). Specifically, NLK-OE shifted the RKO cells from G1 phase towards $S$ phase. However, this trend was reversed upon NLK RNA $i$ (Figure 4B). Therefore, in RKO cells, NLK negatively regulates cell cycle and promotes the apoptosis.

\section{The correlations between the expressions of XIST, miR-92b-3p and NLK in the context of CRC}

There was an interesting pattern of differential expression among XIST, miR-92b-3p, and NLK emerging from the CRC clinical samples under the study. Specially, XIST exhibited higher expression in the CRC tissues than in the adjacent tissues whereases the opposite pattern appeared with miR-92b-3p $(P<0.05)$ (Figure 5A).

We then explored the functional relevance of this pattern to CRC in RKO cells. In response to XIST downregulation via RNA $i$, the expression of miR-92b-3p was significantly upregulated while NLK expression was suppressed (Figure 5B). When the inhibitor of miR-29b was applied, the expressions of XIST and NLK were both increased (Figure 5C). 

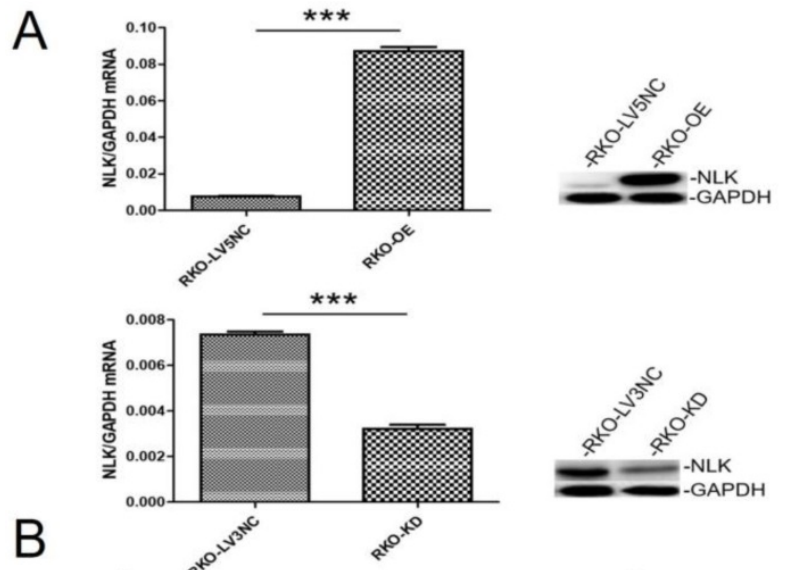

B

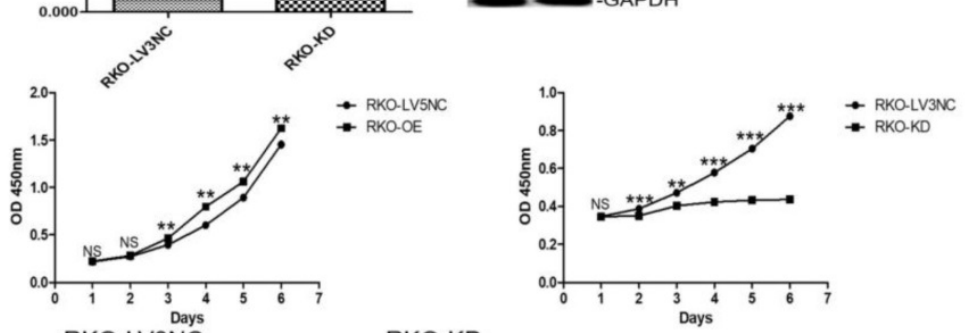

C

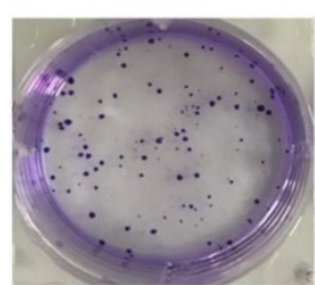

RKO-LV5NC

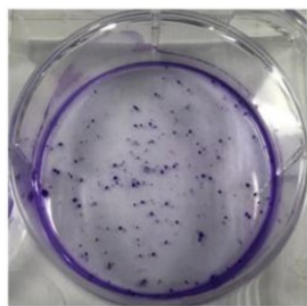

RKO-LV5NC

D

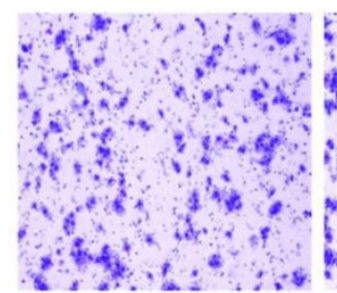

RKO-LV3NC

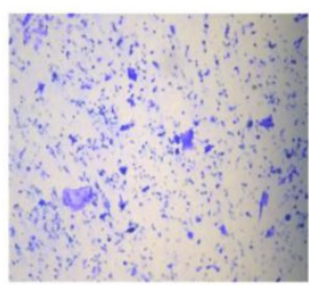

RKO-KD

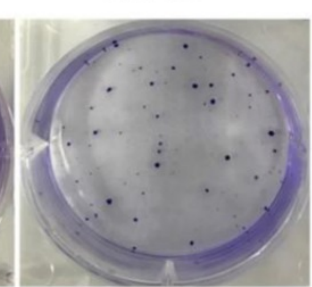

RKO-OE

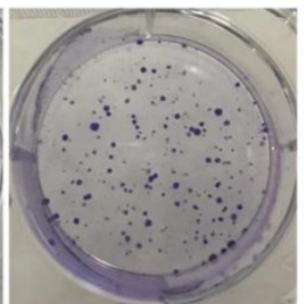

RKO-OE

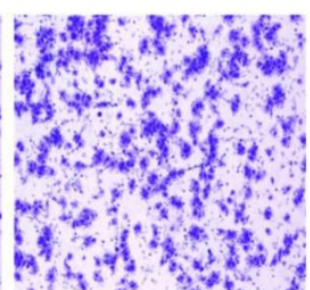

RKO-KD

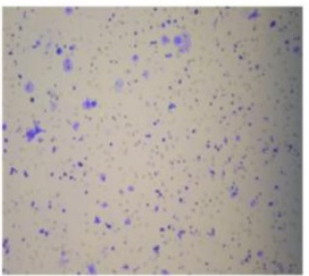

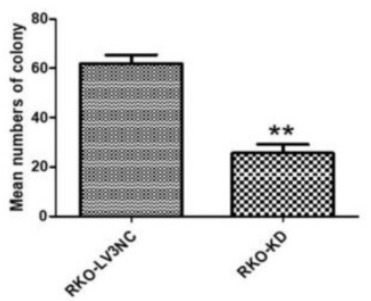
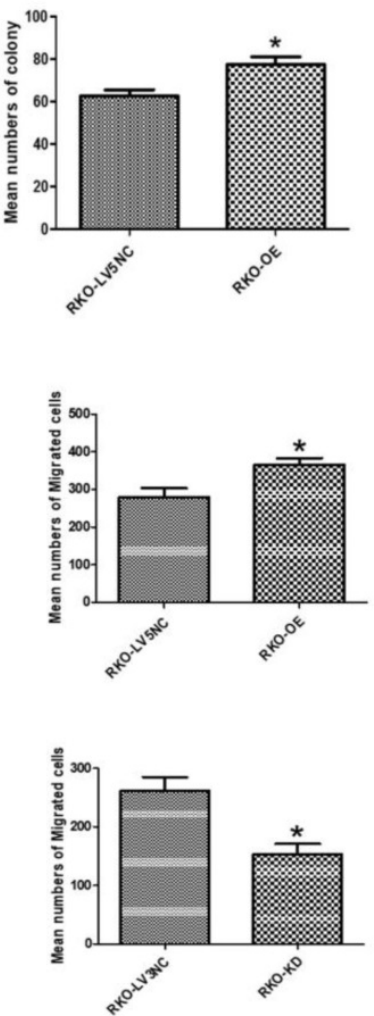

Figure 3. The expression of NLK affects the proliferation and migration of CRC cells in vitro. (A) Knockdown and overexpression of NLK were confirmed by qRT-PCR (left panel) and western blot (right panel) in RKO cells. ***P<0.001. (B) The cell counting assay was performed to measure the proliferation of RKO cells transfected with LV5 compared with those transfected with LV5 NC or shRNA-LV3 and LV3 NC. OD450 values were compared at the indicated time points, $* * P<0.01$, $* * * P<0.001$. (C) Representative images and quantification of the colony forming assay in RKO cells transfected with LV5 or LV5 NC and LV3 or LV3 NC, $* * P<0.01$, $* P<0.05$. (D) Transwell assay was performed to determine the effect of NLK overexpression or knockdown on the migration ability of RKO cells. $* P<0.05$. RKO-OE, RKO cells with overexpression of NLK; RKO-KD, RKO cells with NLK knockdown; q RT-PCR, quantitative real-time polymerase chain reaction. 
A
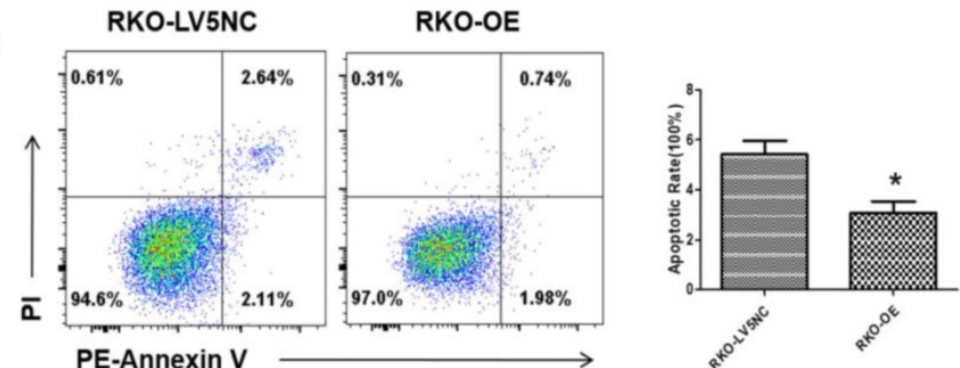

RKO-LV3NC

RKO-KD
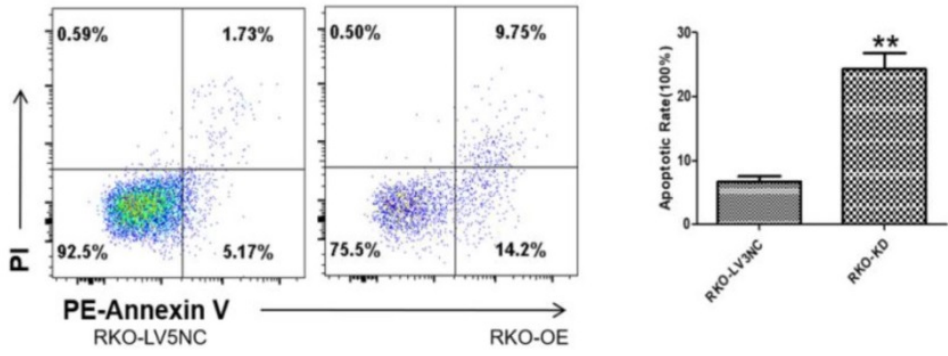

B
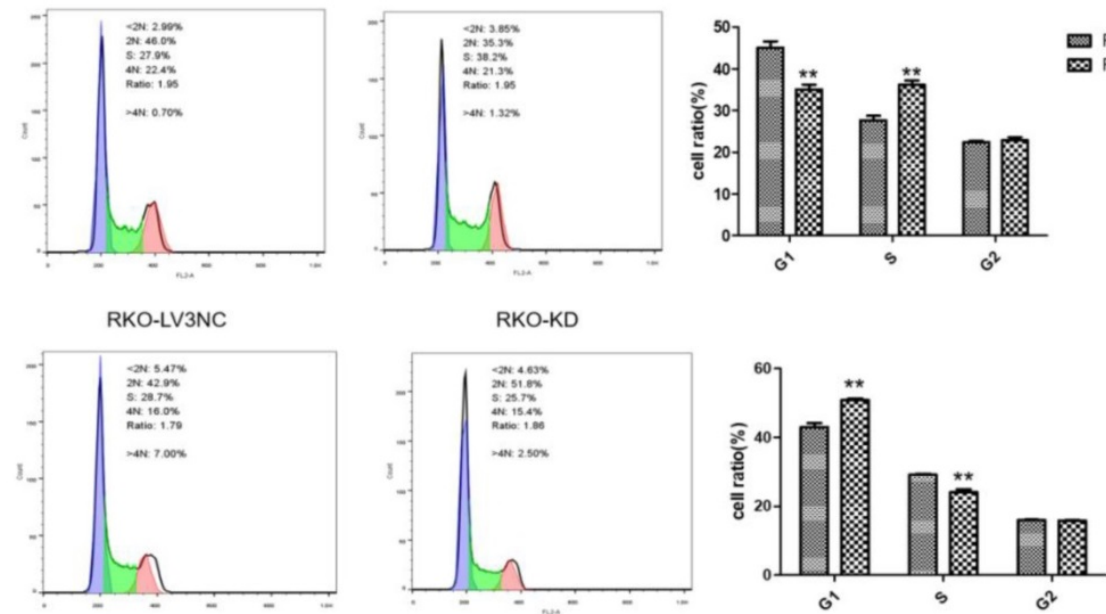

RKO-LV3NC RKO-KD

Figure 4. NLK is required for the cell cycle and apoptosis of RKO cells. Flow cytometry was used to detect the apoptosis (A) and cell cycle (B) of RKO cells transfected with LV5 or LV5 NC and shRNA LV3 or LV3 NC. *P<0.05, **P<0.01, **P<0.01. All results were analyzed by Flowjo Software.

\section{XIST directly binds miR-29b-3p and NLK is a target of miR-29-3p}

We further tested whether interlinked expressions of XIST and miR-92b-3p or miR-92b-3p and NLK is mediated by direct interactions. We established a luciferase-based reporter system in which was to measure the influence of upstream stimulation on the transcription level of downstream target genes, that is, the influence on the mRNA expression level of target genes. First, miR-92b-3p mimics/NC and Report-XIST-wt/mut were co-transfected into $293 \mathrm{~T}$ cells, the fluorescent intensity was measured for the expression of ectopic XIST 24 hours post transfection. As shown in Figure 6A, the expression of XIST was significantly suppressed by the presence of miR-92b-3p, which was abolished when specific mutations were introduced into XIST.
This suggests a direct binding between XIST and miR-92b-3p.

Next, we focused on the relationship between NLK and miR-92b-3p. Similarly, miR-92b-3p suppressed the expression of NLK, and disturbing the potential binding between two disrupted this regulatory relationship (Figure $6 \mathrm{~B}$ ).

\section{Discussion}

Among all cancers, the incidence of CRC ranks third, and the mortality rate ranks second wordwide. In the last 10 years, the mortality and morbidity of CRC in China have increased [31]. Many patients are however only diagnosed when CRC arrives at middle and late stages, and one of key factors for such unfortunate is the lack of specific and sensitive molecular markers for early diagnosis [32]. 
A
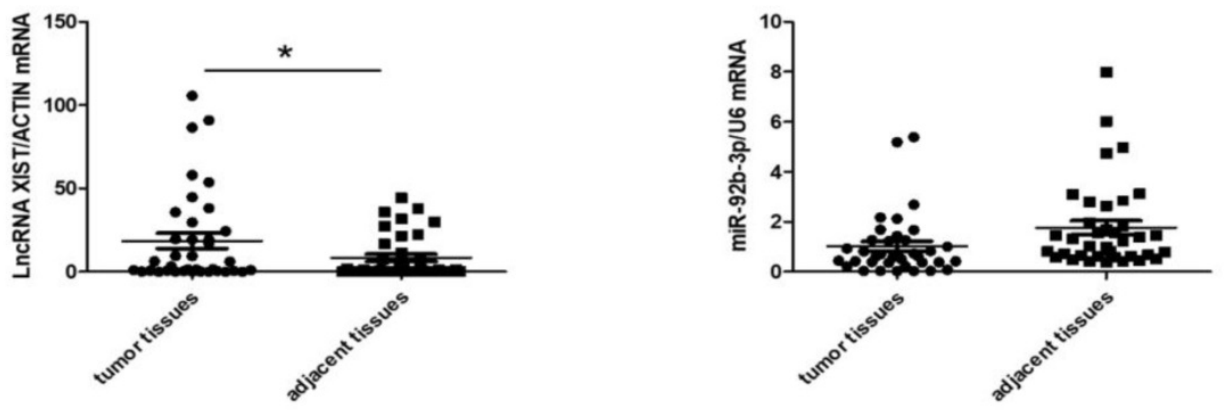

B
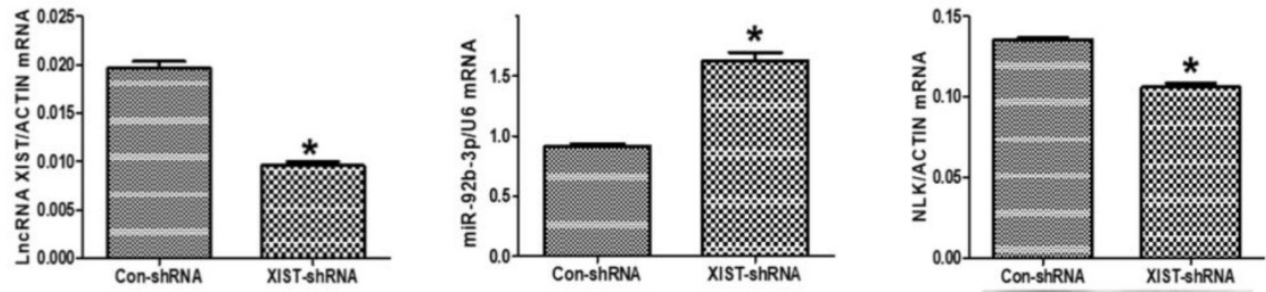

C
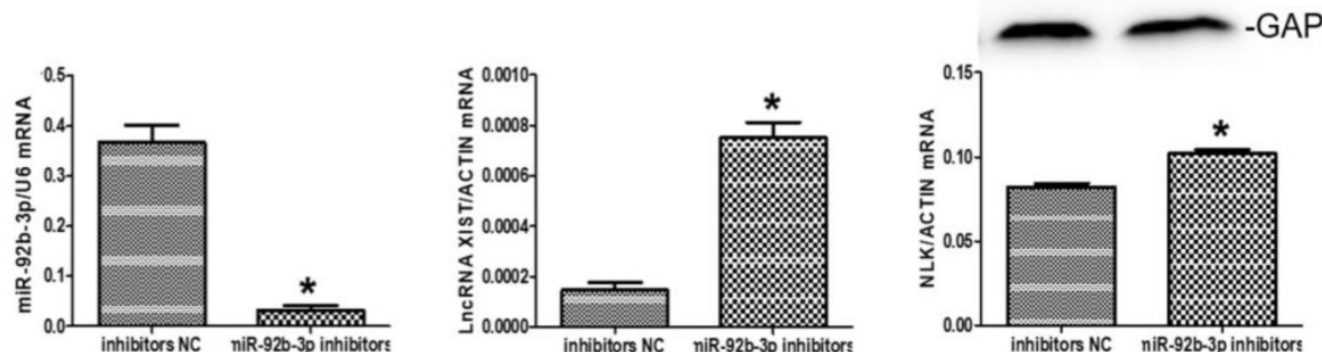

Figure 5. The expressions of LncRNA XISTXIST, miR-92b-3p, and NLK are interlinked. (A) The mRNA expression levels of LncRNA XISTXIST and miR-92b-3p were determined using quantitative real-time polymerase chain reaction in tumor and adjacent tissues. $* P<0.05$. (B) qRT-PCR and western blot were used to examine the mRNA and protein expression of miR-92b-3p and NLK following transfection with XIST-shRNA and the negative control. $* P<0.05$. (C) $q R T-P C R$ was used to examine the mRNA and protein expression of LncRNA XISTXIST and NLK following transfection with miR-92b-3p inhibitors and the negative control. $* P<0.05$.
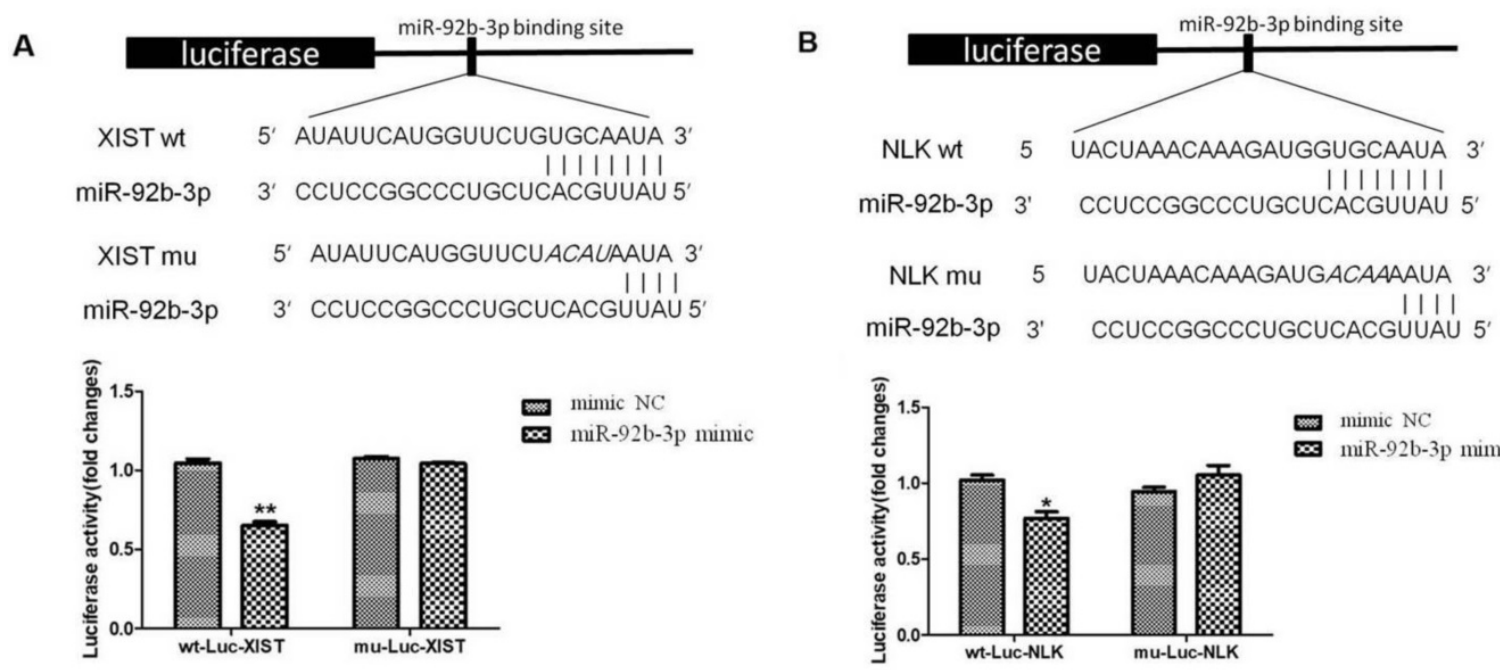

Figure 6. miR-92b-3p regulates XIST and NLK expressions via directly binding to their mRNAs. (A) The wild type and mutated miR-92b-3p binding sites in the XIST 3'-UTR are shown in the upper panel. miR-92b-3p mimic and luciferase constructs were co-transfected into 293 T cells. Luciferase activity of wild type and mutant Luc XIST are shown in the lower panel. (B)The wild type and mutated miR-92b-3p binding sites in the NLK 3'-UTR are shown in the upper panel. Luciferase activity of wild type and mutant Luc NLK are shown in the lower panel. $P<0.05$ vs. control group.

In the past three decades, an increasing number of SNPs have been confirmed to be associated with CRC risk through extensive genome-wide association studies and candidate gene association studies [33]. Particularly, the intronic polymorphic variants can confer susceptibility to diseases as a variety of functional elements are located in introns, ranging from the splicing sites, enhancers, silencers, to cis-acting RNA elements, thereby likely exerting great impact on the target gene expression [34]. Among 
them is the case of NLK, located on human chromosome 17p12. rs2125846 is located in the sixth intron of the NLK gene and is given rise by a A-G base substitution occurs at position 2364 (2364A/G).

Here, we identified a new susceptibility locus, rs2125846, for CRC risk located within an intron of the human NLK gene in a Chinese population. It was statistically related to the tumor size of CRC, the depth of CRC infiltration, and whether the patient had a history of alcohol consumption. We think there are two possible explanations for this association. First, the sample size was insufficient and mainly focused on the Han population in Anhui Province. However, more comprehensive CRC-related risk factors and common clinicopathological features shall be included along with enlarging sample sizes in future work.

NLK has been proposed as a tumor suppressor of therapy interest [35]. We found its expression is specifically related to the onset of CRC. Interestingly, it had been shown that NLK overexpression was related to unfavorable clinicopathological parameters such as advanced TNM stage, poor differentiation, lymph node and distant metastasis, and higher recurrence rates [15]. Our results confirm that NLK expression affect the cell viability in CRC (Figure 3). We speculate that NLK may play a key role in regulating the carcinomagenesis and progression of CRC. To confirm this prediction, subsequent experiments investigated the changes in the biological behavior of CRC cells following NLK overexpression or silencing.

We showed that NLK expression regulates the proliferation ability \& migratory ability of RKO cells (Figure 4). One aspect is linked to the cell cycle progression, in which NLK downregulation led to an accumulation of cells at G1 phase, consistent with the previous report [14]. The proportion of apoptotic cells was decreased after overexpression of NLK but increased after NLK downregulation, which was consistent with the results of cell cycle analysis. The cell cycle disorder caused by the cells in G1 causes the initiation of apoptosis procedures, which in turn causes an increase in apoptotic cells. Specifically, cells were arrested in the G1 phase after downregulation of the NLK gene, which led to an increased number of apoptotic cells.

LncRNAs participate in various biological processes by regulating gene expression at the transcriptional, post-transcriptional, and epigenetic levels [36]. Recently, LncRNAs have been proposed as miRNAs sponges in the context of diseases and their abnormal expressions are associated with certain cancers [18,37]. XIST, the key regulatory factor of mammalian $\mathrm{X}$ inactivation, is also linked to various tumors and related medical conditions [38-40]. More importantly, XIST was shown to be significantly upregulated in CRC tissues and cell lines, being related to poor prognosis [41]. We confirmed here that XIST is upregulated in CRC tissues.

MiR-92b was first reported to be overexpressed in primary brain tumors [42], and then several studies further clarified its carcinogenic effects in glioblastoma [26], non-small cell lung cancer [43], bladder cancer [44], and cholangiocarcinoma [45]. In contrast, miR-92b exhibits anti-tumor activity in many gastrointestinal cancers, such as pancreatic cancer [46] and esophageal squamous cell carcinoma [47]. The level of miR-92b in circulating exosomes was significantly higher in healthy controls compared with CRC patients, especially in those with TNMII stage [48]. In this study, the expression of miR-92b-3p in CRC was found lower than that in the normal adjacent intestinal tissues. However, no clear relationship can be established between mir-92b-3p expression and clinical parameters included, likely due to the limited sample size.

We demonstrated that XIST acts directly on the miR-92b-3p transcript and regulates its expression. In a similar way, miR-92b-3p regulates the expression of NLK. Overall data suggested a possible role ceRNA in CRC cells, in which the XIST/miR-92b-3p/NLK signaling axis could play critical roles, therefore possessing potential therapeutic values.

\section{Abbreviations}

CRC: colorectal cancer; NLK: Nemo-like kinase; SNP: single nucleotide polymorphism; ncRNA: noncoding RNA; LncRNA: Long-stranded non-coding RNA; ceRNA: competitive endogenous RNA; miR: microRNA; IHC: Immunohistochemistry; qRT-PCR: Quantitative real-time polymerase chain reaction; TUNEL: TdT-mediated dUTP Nick-End Labeling; OR: odds ratio.

\section{Acknowledgements}

This study was supported by Anhui Medical University Scientific Research Fund (Grant No. 2020xkj165 and 2020xkj220), the Natural Science Foundation of the Anhui Higher Education Institutions of China (Grant No. KJ2019A0260) and the National Natural Science Foundation of China (Grant No. 81302150 and 81570419).

\section{Ethics statement}

This study protocols were approved by the Ethics Committee of Anhui Medical University. 


\section{Author Contributions}

X-WC and H-QZ contributed to the conception and design of the experiments, gave final approval, and agreed to be accountable for all aspects of work ensuring integrity and accuracy. X-YC, Y-FZ and $Y-F W$ drafted and critically revised the manuscript, $\mathrm{X}-\mathrm{YC}, \mathrm{Y}-\mathrm{FZ}$ and T-TC completed the human and cell experiments. X-YC, Y-FZ and Y-FW contributed to the analysis.

\section{Competing Interests}

The authors have declared that no competing interest exists.

\section{References}

1. Zhou R, Zhang JW, Zeng DQ, et al. Immune cell infiltration as a biomarker for the diagnosis and prognosis of stage I-III colon cancer. Cancer Immunol Immunother. 2019;68(3):433-442.

2. Koncina E, Haan S, Rauh S, et al. Prognostic and Predictive Molecular Biomarkers for Colorectal Cancer: Updates and Challenges. Cancers(Basel). 2020;12(2):319.

3. Ma XY, Zhang B, Zheng W. Genetic variants associated with colorectal cancer risk:comprehensive research synopsis, meta-analysis, and epidemiological evidence. Gut. 2014;63(2):326-336.

4. Shen CQ, Yan TT, Wang ZH, et al. Variant of SNP rs1317082 at CCSlnc362 (RP11-362K14.5) creates a binding site for miR-4658 and diminishes the susceptibility to CRC. Cell Death Dis.2018; 9(12):1177.

5. Brott BK, Pinsky BA, Erikson RL. Nlk is a murine protein kinase related to Erk/MAP kinases and localized in the nucleus. Proc Natl Acad Sci U S A. 1998;95(3):963-968

6. Choi KW, Benzer S. Rotation of photoreceptor clusters in the developing Drosophila eye requires the nemo gene. Cell. 1994;78(1):125-136.

7. Lei L, Wang Y, Zheng YW, et al. Overexpression of Nemo-like Kinase Promotes the Proliferation and Invasion of Lung Cancer Cells and Indicates Poor Prognosis. Curr Cancer Drug Targets. 2019;19(8):674-680.

8. Shen N, Duan XH, Wang XL, et al. Effect of NLK on the proliferation and invasion of laryngeal carcinoma cells by regulating CDCP1. Eur Rev Med Pharmacol Sci. 2019;23(14):6226-6233.

9. Yang WN, Gu LN, Yang C, et al. Expression of Nemo-like kinase in cervical squamous cell carcinoma: a clinicopathological study. Onco Targets Ther. 2018;11: 743-749.

10. Shi C, Xu LQ, Tang ZY, et al. Knockdown of Nemolike kinase promotes metastasis in nonsmallcell lung cancer. Oncol Rep. 2019;42(3):1090-1100.

11. Huang $Y Q$, Jiang $Y$, Lu WQ, et al. Nemo-like kinase associated with proliferation and apoptosis by c-Myb degradation in breast cancer. PLoS One. 2013;8(7):e69148.

12. Zhang YQ, Peng $C, W u$ G, et al. Expression of NLK and its potential effect in ovarian cancer chemotherapy. Int $J$ Gynecol Cancer. 2011;21(8):1380-1387.

13. Stevens KN, Kelemen LE, Wang XS, et al. Common variation in Nemo-like kinase is associated with risk of ovarian cancer. Cancer Epidemiol Biomarkers Prev. 2012;21(3):523-528.

14. Li SZ, Zeng F, Li J, et al. Nemo-like kinase (NLK) primes colorectal cancer progression by releasing the E2F1 complex from HDAC1. Cancer Lett.2018;431: 43-53.

15. Zhang W, He J, Du Y, et al. Upregulation of nemo-like kinase is an independent prognostic factor in colorectal cancer. World I Gastroenterol. 2015;21(29):8836-8847.

16. Han Y, Kuang YT, Xue XF, et al. NLK, a novel target of miR-199a-3p, functions as a tumor suppressor in colorectal cancer. Biomed Pharmacother. 2014;68(5):497-505.

17. Djebali S, Davis CA, Merkel A, et al. Landscape of transcription in human cells. Nature.2012; 489(7414):101-108.

18. Sun BY, Liu CX, $\mathrm{Li} \mathrm{H}$, et al. Research progress on the interactions between long non-coding RNAs and microRNAs in human cancer. Oncol Lett. 2020;19(1):595-605.

19. Yang Q, Tang YY, Tang CX, et al. Diminished LINC00173 expression induced miR-182-5p accumulation promotes cell proliferation, migration and apoptosis inhibition via AGER/NF-kappaB pathway in non-small-cell lung cancer. Am I Transl Res. 2019;11(7):4248-4262.

20. Li XY, Zhou LY, Luo H, et al. The long noncoding RNA MIR210HG promotes tumor metastasis by acting as a ceRNA of miR-1226-3p to regulate mucin-1c expression in invasive breast cancer. Aging (Albany NY). 2019;11(15):5646-5665

21.Seitz H. Redefining microRNA targets. Curr Biol. 2009;19(10):870-873.

22. Salmena L, Poliseno L, Tay Y,et al. A ceRNA hypothesis: the Rosetta Stone of a hidden RNA language?. Cell. 2011;146(3):353-358.

23. Khoury S, Tran N. Circulating microRNAs: potential biomarkers for common malignancies. Biomark Med. 2015;9(2),131-151.

24. Wang LY, Cho KB, Li Y, et al. Long Noncoding RNA (lncRNA)-Mediated Competing Endogenous RNA Networks Provide Novel Potential Biomarkers and Therapeutic Targets for Colorectal Cancer. Int J Mol Sci. 2019;20(22):5758.

25. Liu ZM, Diep C, Mao TT, et al. MicroRNA-92b promotes tumor growth and activation of NF-kappaB signaling via regulation of NLK in oral squamous cell carcinoma. Oncol Rep. 2015;34(6):2961-2968.

26. Wang K, Wang X, Zou J, et al. miR-92b controls glioma proliferation and invasion through regulating Wnt/beta-catenin signaling via Nemo-like kinase. Neuro Oncol. 2013;15(5):578-588.

27. Zhuang LK, Yang YT, Ma X, et al. MicroRNA-92b promotes hepatocellular carcinoma progression by targeting Smad7 and is mediated by long non-coding RNA XIST. Cell Death Dis. 2016;7(4): e2203.

28. Liu Y, Bodmer WF. Analysis of P53 mutations and their expression in 56 colorectal cancer cell lines. Proc Natl Acad Sci U S A. 2006;103(4):976-981.

29. Shen HX, Xing C, Cui K et al. MicroRNA-30a attenuates mutant KRAS-driven colorectal tumorigenesis via direct suppression of ME1.Cell Death Differ. 2017;24(7):1253-1262.

30. Ronen J, Hayat S, Akalin A. Evaluation of colorectal cancer subtypes and cell lines using deep learning. Life Sci Alliance. 2019;2(6):e201900517.

31. Bray F, Ferlay J, Soerjomataram I,et al. Global cancer statistics 2018: GLOBOCAN estimates of incidence and mortality worldwide for 36 cancers in 185 countries. CA Cancer J Clin. 2018;68(6):394-424.

32. Center MM, Jemal A, Smith RA, et al. Worldwide variations in colorectal cancer. CA Cancer J Clin. 2009;59(6):366-378

33. Wen J, Xu Q, Yuan Y. Single nucleotide polymorphisms and sporadic colorectal cancer susceptibility: a field synopsis and meta-analysis. Cancer Cell Int. 2018;18:155.

34. Millar DS, Horan M, Chuzhanova NA et al Characterisation of a functional intronic polymorphism in the human growth hormone (GH1) gene. Hum Genomics. 2010;4(5):289-301.

35. Huang $Y$, Yang $Y, H e ~ Y$, et al. The emerging role of Nemo-like kinase (NLK) in the regulation of cancers. Tumour Biol. 2015;36(12):9147-9152.

36. Fan QW, Liu BR. Comprehensive analysis of a long noncoding RNA-associated competing endogenous RNA network in colorectal cancer. Onco Targets Ther, 2018;11: 2453-2466.

37. Wang JY, Yang $\mathrm{Y}, \mathrm{Ma} \mathrm{YJ}$, et al. Potential regulatory role of IncRNA-miRNA-mRNA axis in osteosarcoma. Biomed Pharmacother. 2020;121: 109627.

38. Wang SL, Li GZ. RETRACTED ARTICLE:LncRNA XIST inhibits ovarian cancer cell growth and metastasis via regulating miR-150-5p/PDCD4 signaling pathway. Naunyn Schmiedebergs Arch Pharmacol. 2021;394(4):763.

39. Cui CL, Li YN, Cui XY,et al. IncRNA XIST promotes the progression of laryngeal squamouscell carcinoma by sponging miR144 to regulate IRS1 expression. Oncol Rep. 2020;43(2):525-535.

40. Shi JF, Tan SL, Song LM, et al. LncRNA XIST knockdown suppresses the malignancy of human nasopharyngeal carcinoma through XIST/miRNA-148a-3p/ADAM17 pathway in vitro and in vivo. Biomed Pharmacother. 2020;121: 109620.

41. Chen DL, Chen LZ, Lu YX, et al. Long noncoding RNA XIST expedites metastasis and modulates epithelial-mesenchymal transition in colorectal cancer. Cell Death Dis. 2017;8(8), e3011.

42. Nass D, Rosenwald S, Meiri E, et al. MiR-92b and miR-9/9* are specifically expressed in brain primary tumors and can be used to differentiate primary from metastatic brain tumors. Brain Pathol. 2009;19(3):375-383.

43. Lei L, Huang YP, Gong WR. Inhibition of miR-92b suppresses nonsmall cell lung cancer cells growth and motility by targeting RECK. Mol Cell Biochem. 2014;387(1-2):171-176.

44. Huang J, Wang B, Hui K, et al. miR-92b targets DAB2IP to promote EMT in bladder cancer migration and invasion. Oncol Rep. 2016;36(3):1693-1701

45. Zhou MH, Zhou HW, Liu $M$, et al. The role of miR-92b in cholangiocarcinoma patients. Int J Biol Markers. 2018;33(3):293-300.

46. Long MM, Zhan M, Xu SW, et al. miR-92b-3p acts as a tumor suppressor by targeting Gabra3 in pancreatic cancer. Mol Cancer. 2017;16(1):167.

47. Cheng LN, Yang F, Zhou BX, et al. RAB23, regulated by miR-92b, promotes the progression of 7esophageal squamous cell carcinoma. Gene. 2016;595(1):31-38. 
48. Min L, Chen L, Liu S, et al. Loss of Circulating Exosomal miR-92b is a Novel Biomarker of Colorectal Cancer at Early Stage. Int J Med Sci. 2019;16(9):1231-1237. 